\title{
Time-Domain Hybrid Global-Local Prediction of Guided Waves Interaction with Damage
}

\author{
Matthieu Gresil ${ }^{1, a}$ and Victor Giurgiutiu ${ }^{1, b}$ \\ ${ }^{1}$ University of South Carolina, 300 Main Street, Columbia, SC 29208, USA \\ amatthieu@engr.sc.edu (corresponding author) \\ bvictorg@.sc.edu
}

\begin{abstract}
Keywords: Guided wave, finite element method, structural health monitoring, piezoelectric wafer active sensor, hybrid global-local.
\end{abstract}

\begin{abstract}
This paper presents a hybrid finite element and analytical method to predict the 1-D guided wave propagation interaction with damage for nondestructive evaluation (NDE) and structural health monitoring (SHM) application. The finite element mesh is used to describe the region around the damage (defects or flaws). In contrast to other hybrid models developed elsewhere, the interaction between Lamb waves and defects is computed in the time domain using the explicit solver of the commercial finite element method (FEM) software ABAQUS. Analytical methods can perform efficient modeling of wave propagation but are limited to simple geometries. Realistic structures with complicated geometries are usually modeled with the FEM. However, to obtain an accurate wave propagation solution at ultrasonic frequencies is computationally intensive and may become prohibitive for realistic structures. In response to today's complex cases not covered by the simulation tools available, we aim to develop an efficient and accessible tool for SHM applications. This tool will be based on a hybrid coupling between analytical solutions and time domain numerical codes. Lamb wave interaction with a notch is investigated by using this method, and the results obtained are with respect to transmission, reflection and mode conversion. Because of the symmetric mode shape, S0 is more sensitive to the shallow notch than A0. By making use of the fact that the reflection increases with increase in notch depth and mode conversion are maximized when the notch is around half through the thickness of the plate, the reflection and conversion coefficients can be used to characterize the depth of the notch.
\end{abstract}

\section{Introduction}

Many authors have already investigated the interaction of Lamb waves with a single defect like crack, notch, or circular cavity. Some of them used analytical [1] or semi-analytical [2] solutions, whereas others chose a finite element, a boundary element, or spectral element method [3-10]. Analytical or semi-analytical solutions can be used when the geometry of the defect is regular and when the problem presents symmetries. Finite or boundary element modeling (FEM/BEM) allows studying the interaction of Lamb modes with irregular defects but require respecting spatial and temporal discretization norms which can cause computational problems at high frequency-thickness product values. In addition, to obtain accurate wave propagation FEM/BEM solution at ultrasonic frequencies is computationally intensive and may become prohibitive for realistic structures.

A hybrid formulation can be used wherein the finite element method is employed to model small regions near the defect whereas regions away from the defect are modeled using a suitable set of analytical wave functions. Goetschel et al. [11] developed a global-local finite element formulation for modeling axisymmetric scattering of a steady, compressive, incident elastic wave in a homogeneous, isotropic host medium with an axisymmetric inclusion. The method was later applied to model wave interaction with defected lap-shear joints [12], as well as notches in plates [13].

Many structural components are complex in either their geometry or their material properties. In this case, theoretical wave solutions for the global portion are either nonexistent or hard to determine. The Semi Analytical Finite Element (SAFE) method can help handle these cases because of its ability to extract modal solutions of complex structures in a computationally efficient manner 
[14-17]. Sabra et al. [18] demonstrated the application of the SAFE-aided hybrid formulation to the detection of holes in aluminum plates. Srivastava [19] extends the global-local approach to model notches in aluminum plates and delamination-like defects in composite panels.

In contrast to the above hybrid formulations, this paper calculates the interaction between Lamb waves and defects in the time domain. This approach is implemented in three steps using propriatary analytical software (WaveFormRevealer) and commercial FEM software. The first step of this method uses the WaveFormRevealer software developed for this application. The second step uses the output from the WaveFormRevealer software as inputs into the FEM model. The third step of our method uses again the WaveFormRevealer software to transfer the output from the FEM local damage to the receiver transducer placed in the global region away from damage. The FEM output serves as input into the Global region. This hybrid global-local-global approach is compared with the analytical solution and experimental results.

\section{Fundamental of Lamb waves}

Guided waves (Lamb waves) travel at long distances in thin wall structures. Guided waves are typically transmitted and received using piezoelectric wafer active sensors (PWAS) [25], and are used to detect defects and damage in thin wall structures. Two types of guided waves propagate: symmetric waves and antisymmetric waves. Each of these wave types propagates with multiple modes.

By solving a boundary value problem for a free plate or by considering the reflection of waves at plate boundaries, the Rayleigh-Lamb frequency equations (dispersion equations) can be found [2023]. For a uniform traction-free isotropic plate, the equation for symmetric modes is

$$
\frac{\tan (q h)}{\tan (p h)}=-\frac{4 k^{2} p q}{\left(q^{2}-k^{2}\right)^{2}}
$$

For antisymmetric modes, we have

$$
\frac{\tan (q h)}{\tan (p h)}=-\frac{\left(q^{2}-k^{2}\right)^{2}}{4 k^{2} p q}
$$

where

$$
p^{2}=\frac{\omega^{2}}{c_{L}^{2}}-k^{2}, \quad q^{2}=\frac{\omega^{2}}{c_{T}^{2}}-k^{2}, \quad k=\frac{\omega}{c_{p}}
$$

The notations $h, k, c_{L}, c_{T}, c_{p}, \omega$ signify the half plate thickness, wave number, velocities of longitudinal and transverse modes, phase velocity, wave circular frequency, respectively.

The phase velocity is found numerically by solving the roots of the dispersion equation as a function of material properties, frequency, and material thickness. Group velocity dispersion curves are found from the phase velocity [23].

Phase and group velocity dispersion curves for a $3.2 \mathrm{~mm}$-thick aluminum plate are shown in Figure 1 , where longitudinal and transverse velocities of the plate are equal to $c_{L}=6211 \mathrm{~m} / \mathrm{s}$, and $c_{T}=3129 \mathrm{~m} / \mathrm{s}$, respectively. These curves were generated and plotted using our propriatary software "Wavescope" [24]. Figure 1 shows the dispersion curves of the first two symmetric and antisymmetric modes. At low frequencies, the wave velocity of the first symmetric mode (S0) is nearly non-dispersive, while the wave velocity of the first antisymmetric mode (A0) is highly dispersive. At higher frequencies, group velocity of both A0 and S0 approaches the Rayleigh wave velocity, $c_{R} \approx 2900 \mathrm{~m} / \mathrm{s}$. 

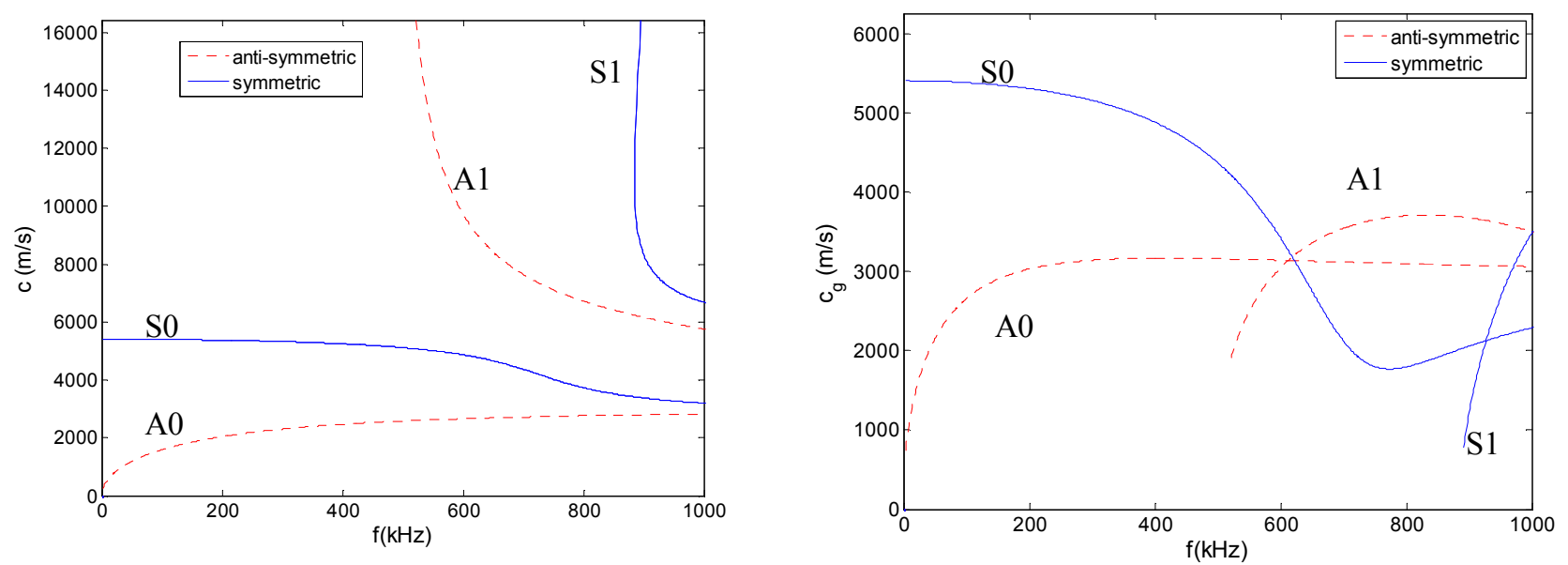

Figure 1: Dispersion curves for 3.2-mm thick aluminum plate: (a) phase velocity dispersion curves; (b) group velocity dispersion curves.

\section{Analytical model for the global domain}

PWAS transducers are the enabling technology for active and passive SHM systems [25]. PWAS couples the electrical and mechanical effects through the tensorial piezoelectric constitutive equations

$$
S_{i j}=s_{i j k l}^{E} T_{k l}+d_{k i j} E_{k}, \quad D_{j}=d_{j k l} T_{k l}+\varepsilon_{j k}^{T} E_{k}
$$

where, $S_{i j}$ is the mechanical strain; $T_{k l}$ is the mechanical stress; $E_{k}$ is the electrical field; $D_{j}$ is the electrical displacement; $s_{i j k l}^{E}$ is the mechanical compliance of the material measured at zero electric field $E=0, \varepsilon_{j k}^{T}$ is the dielectric permittivity measures at zero mechanical stress $T=0$, and $d_{k i j}$ represents the piezoelectric coupling effect. PWAS utilize the $d_{31}$ coupling between in-plane strain and transverse electric field. A 7-mm diameter, 0.2mm thick, PWAS weights around $78 \mathrm{mg}$ and costs around \$1. PWAS transducers are lightweight and inexpensive and hence can be deployed in large numbers on the monitored structure.

PWAS transducers can serve several purposes [25]: (a) high-bandwidth strain sensors; (b) highbandwidth wave exciters and receivers; (c) resonators; (d) embedded modal sensors with the electromechanical impedance spectroscopy method. By application types, PWAS transducers can be used for (i) active sensing of far field damage using pulse-echo, pitch-catch, and phased-array methods, (ii) active sensing of near field damage using the high frequency electro-mechanical impedance spectroscopy (EMIS) and thickness gage mode, and (iii) passive sensing of damagegenerating events through detection of low-velocity impacts and acoustic emission at the tip of advancing cracks.

The analytical modeling of the pitch-catch process between two PWAS transducers separated by a distance $x$ is carried out in frequency domain in four steps [26-28]:

- Fourier transform the time-domain excitation signal $V_{e}(t)$ into the frequency domain spectrum, $\tilde{V}_{e}(\omega)$;

- Calculate the frequency-domain structural transfer function at the receiver location, $G(x, \omega)$;

- Multiply the structural transfer function by frequency-domain excitation signal to obtain the frequency domain signal at the receiver, i.e., $\tilde{V}_{r}(x, \omega)=G(x, \omega) \cdot \tilde{V}_{e}(\omega)$;

- Perform inverse Fourier transform to obtain the time-domain receiver signal, $V_{r}(x, t)=\operatorname{IFFT}\left\{\tilde{V}_{r}(x, \omega)\right\}=\operatorname{IFFT}\left\{G(x, \omega) \cdot \tilde{V}_{e}(\omega)\right\}$. 
In this paper, the main interest is in symmetric fundamental mode $\left(\mathrm{S}_{0}\right)$ and anti-symmetric fundamental mode $\left(\mathrm{A}_{0}\right)$. For Lamb waves with only two modes $\left(\mathrm{A}_{0}\right.$ and $\left.\mathrm{S}_{0}\right)$ excited, the structure transfer function $G(\omega)$ is given by Eq. (99) of ref. [25], page 327, which gives the in-plane strain at the plate surface as

$$
\begin{gathered}
\left.\varepsilon_{x}(x, t)\right|_{y=d}=-i \frac{a \tau_{0}}{\mu}\left(\sin k^{S} a\right) \frac{N_{S}\left(k^{S}\right)}{D_{S}^{\prime}\left(k^{S}\right)} e^{-i\left(k^{S} x-\omega t\right)} \\
-i \frac{a \tau_{0}}{\mu}\left(\sin k^{A} a\right) \frac{N_{A}\left(k^{A}\right)}{D_{A}^{\prime}\left(k^{A}\right)} e^{-i\left(k^{A} x-\omega t\right)} \\
G(\omega)=S(\omega) e^{-i k^{S} x}+A(\omega) e^{-i k^{A} x} \\
S(\omega)=-i \frac{a \tau_{0}}{\mu}\left(\sin k^{S} a\right) \frac{N_{S}\left(k^{S}\right)}{D_{S}^{\prime}\left(k^{S}\right)}, \quad A(\omega)=-i \frac{a \tau_{0}}{\mu}\left(\sin k^{A} a\right) \frac{N_{A}\left(k^{A}\right)}{D_{A}^{\prime}\left(k^{A}\right)} \\
D_{S}(\omega, d)=\left(k^{2}-\beta^{2}\right)^{2} \cos \alpha d \sin \beta d+4 k^{2} \alpha \beta \sin \alpha d \cos \beta d \\
D_{A}(\omega, d)=\left(k^{2}-\beta^{2}\right)^{2} \sin \alpha d \cos \beta d+4 k^{2} \alpha \beta \cos \alpha d \sin \beta d \\
N_{S}=k \beta\left(k^{2}-\beta^{2}\right) \cos (\alpha d) \sin (\beta d), \quad N_{A}=k \beta\left(k^{2}-\beta^{2}\right) \sin (\alpha d) \cos (\beta d) \\
\alpha^{2}=\frac{\omega^{2}}{c_{p}^{2}}-k^{2}, \quad \beta^{2}=\frac{\omega^{2}}{c_{s}^{2}}-k^{2}
\end{gathered}
$$

where $a$ is the half length of the PWAS, $d$ is the half thickness of the plate, $\tau_{0}$ is the shear stress between PWAS and the plate, $\mu$ is Lame's constant, $k^{S}$ and $k^{A}$ are the wavenumbers for $\mathrm{S}_{0}$ and $\mathrm{A}_{0}$ respectively, $x$ denotes the distance between the two PWAS transducers, $k$ represents the wavenumber for $\mathrm{S}_{0}$ or $\mathrm{A}_{0}$ accordingly, $c_{p}$ and $c_{s}$ are the wave speed for pressure wave and shear wave respectively. In the transfer function, it could be observed that $S(\omega)$ and $A(\omega)$ will determine the amplitude of $\mathrm{S}_{0}$ and $\mathrm{A}_{0}$ mode. In both $S(\omega)$ and $A(\omega)$ terms, there is $\sin \left(k^{S} a\right)$ and $\sin \left(k^{A} a\right)$, which represent the tuning effect.

The wave speed dispersion curve is obtained by solving Rayleigh-Lamb equations (1) and (2), which are transcendental equations that require numerical solution. The usual form of RayleighLamb equations (1) and (2) expressed as

$$
D_{S}(\omega, d)=0 \quad \text { and } \quad D_{A}(\omega, d)=0
$$

After getting the wave speed dispersion curve, the wavenumber for each frequency component i.e. $\xi=\omega / c$ is known. Thus, all the terms involved in the plate transfer function could be solved, and the plate transfer function $G(\omega)$ is obtained. After the plate transfer function $G(\omega)$ is obtained, the excitation signal is Fourier transformed. 


\section{Software development for the global domain}

The analytical model presented in the previous section was used to develop the software program 'WaveFormRevealer' utilizing the Matlab graphical user interface (GUI) environment to predict the waveform for the analytical modeling part of the HGL approach [26, 27]. The GUI of the software is shown in Figure 2.

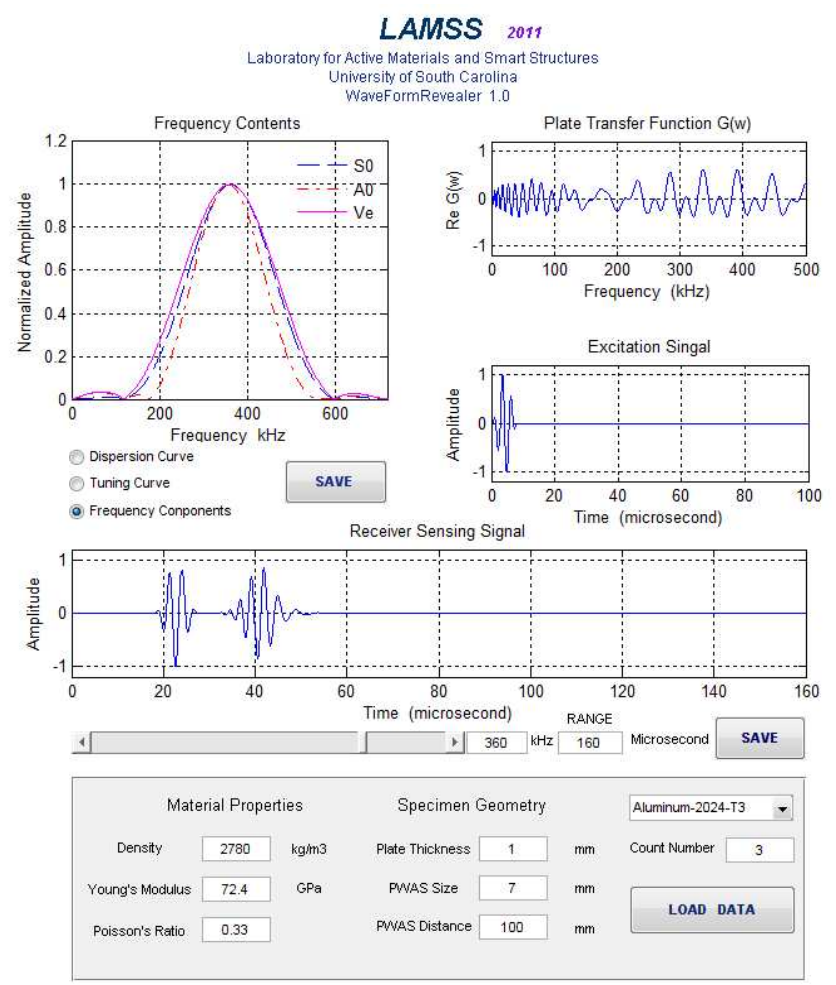

Figure 2: Interface of the analytical software „WaveFormRevealer 1.0“ [27-29].

This software allows users to get the desired analytical signal prediction by inputting material properties, specimen geometry, excitation signal count number, excitation signal frequency, and time range. It can also show a continuous waveform change by clicking on the frequency control slider, which is just like the waveform shown on an oscilloscope when adjusting the excitation signal frequency. The signal range in time domain can be set by entering the 'Range' information.

\section{Finite element model in the local region}

The effectiveness of conventional finite element modeling of elastic waves propagating in structural components has been shown for the case of Lamb waves in free plates in [30, 31]. The FEM package used in the present study, ABAQUS/Explicit, uses an explicit time integration based on a central difference scheme [32]. The stability of the numerical solution is dependent upon the temporal and the spatial resolution of the analysis. The maximum frequency of the dynamic problem, $f_{\max }$, limits both the integration time step and the element size. To avoid numerical instability, ABAQUS/Explicit [32] recommends a stability limit for the integration time step of [31]

$$
\Delta t=\frac{1}{20 f_{\max }}
$$

The size of the finite element mesh, $L_{e}$, is typically derived from the smallest wavelength to be analyzed, $\lambda_{\min }$. For a good spatial resolution, 20 nodes per wavelength are advisable [31]. This condition can be written as

$$
L_{e}=\frac{\lambda_{\min }}{20}
$$


To generate the appropriate Lamb mode ( $\mathrm{S} 0$ or A0), boundary conditions are imposed on the plate. To simulate the time profile of the excitation, a 3-cycle tone burst enclosed in a Hanning window centered on the excitation frequency is used. The plate length is defined to prevent reflections from the edges and is minimized to reduce the computing time.

\section{Experimental set-up}

A test specimen was designed to develop and calibrate the damage-detection methodology using a simple geometry specimen, and also to validate the analytical, and the hybrid global-local results. The aluminum plate specimen was constructed from 3.2-mm-thick 2024-alloy stock in the form of a square plate $(1118 \mathrm{~mm} \times 1118 \mathrm{~mm} \times 3.2 \mathrm{~mm})$. The specimen was instrumented with arrays of $7-\mathrm{mm}$ square and 7-mm circular PWAS (Figure 3). The $(x, y)$ sensor locations are given in Table 1. An HP33120A arbitrary signal generator was used to generate a $150 \mathrm{kHz}$ windowed harmonic-burst excitation to active sensors with a $10 \mathrm{~Hz}$ repetition rate. Under harmonic burst excitation, the transmitter PWAS generates a packet of elastic waves that spread out into the entire plate according to a circular wave front pattern. A Tektronix TDS210 four-channel digital oscilloscope, synchronized with the signal generator, was used to collect the response signals from the receiver PWAS transducers.

The results of the rectangular plate tests were remarkably clear and easy to interpret. Reflections wave packets could be easily separated because the edges of the rectangular plate were far away from the source.

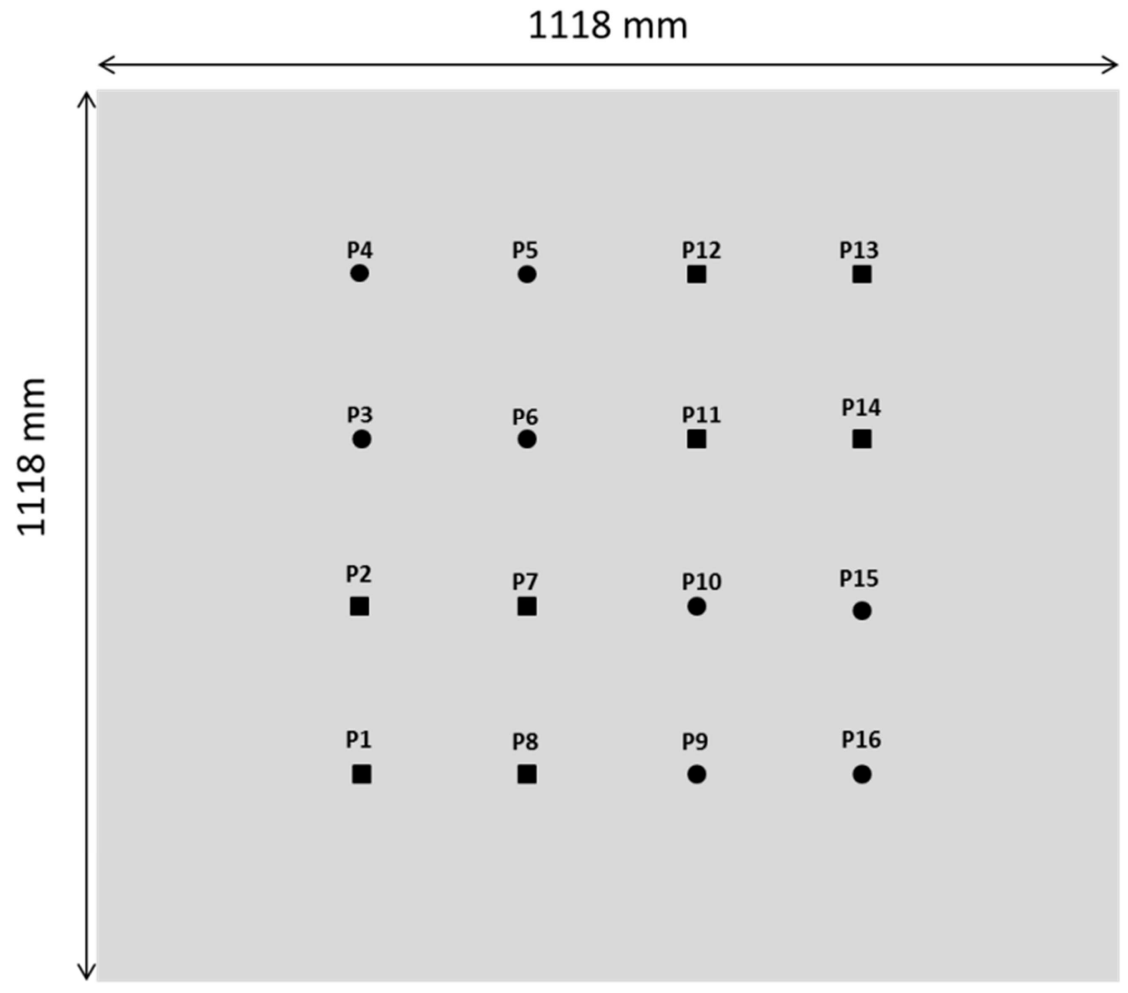

Figure 3: Arrangment of piezoelectric wafer active sensor (PWAS) transducers bonded on the plate specimen.

Table 1: Location of PWAS transducers on the square plate specimen.

\begin{tabular}{|c|c|c|c|c|c|c|c|c|c|c|c|c|c|c|c|c|}
\hline PWAS\# & 1 & 2 & 3 & 4 & 5 & 6 & 7 & 8 & 9 & 10 & 11 & 12 & 13 & 14 & 15 & 16 \\
\hline $\mathbf{x ~ ( m m )}$ & 31 & 31 & 31 & 31 & 51 & 51 & 51 & 51 & 71 & 71 & 71 & 71 & 91 & 91 & 91 & 91 \\
\hline $\mathbf{y}(\mathbf{m m})$ & 31 & 51 & 71 & 91 & 91 & 71 & 51 & 31 & 31 & 51 & 71 & 91 & 91 & 71 & 51 & 31 \\
\hline Square & $\mathrm{X}$ & $\mathrm{X}$ & & & & & $\mathrm{X}$ & $\mathrm{X}$ & & & $\mathrm{X}$ & $\mathrm{X}$ & $\mathrm{X}$ & $\mathrm{X}$ & & \\
\hline Circular & & & $\mathrm{X}$ & $\mathrm{X}$ & $\mathrm{X}$ & $\mathrm{X}$ & & & $\mathrm{X}$ & $\mathrm{X}$ & & & & & $\mathrm{X}$ & $\mathrm{X}$ \\
\hline
\end{tabular}




\section{Hybrid Global-Local Method}

In response to today's most complex cases not covered by the simulation tools available, we aim to develop an efficient and accessible tool simulation for SHM application. This tool is based on a hybrid coupling between analytical solution and numerical codes. We couple global analytical calculation with local FEM analysis to utilize the advantages of both methods and obtain a rapid and accurate simulation method. Figure 4 illustrates this concept, whereby only the local material region close to the defect needs to be FEM modeled whereas the global waveguides to the left and right of the FEM region are modeled analytically with compatibility being ensured at the interface.

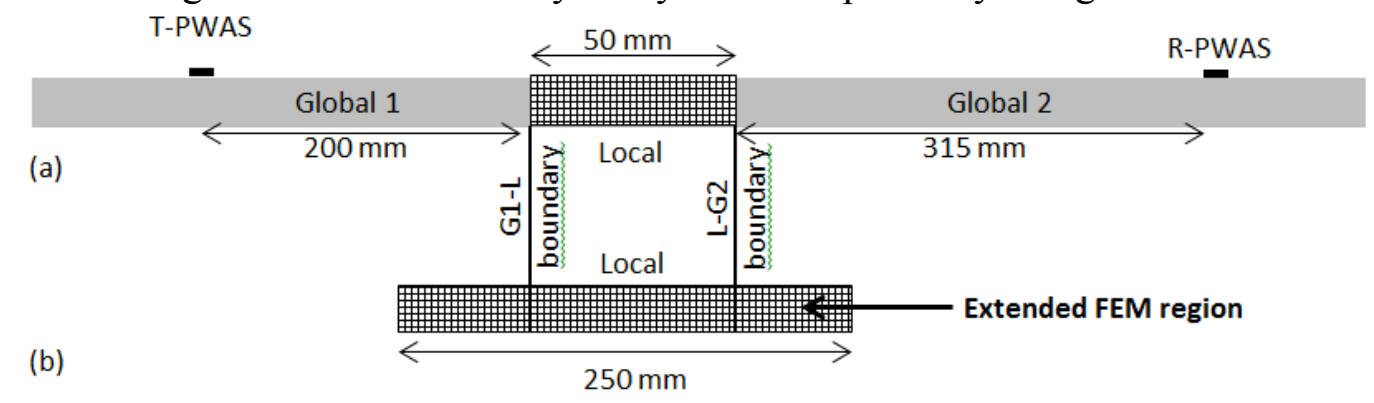

Figure 4: Waveguide problem in 1-D solved by the hybrid global-local (HGL) method in the time domain: (a) overall HGL set-up; (b) FEM discretization including extended regions for reflection a voidance.

The first step of this method consists in using the WaveFormRevealer software to predict analytically the wave propagation in the "Global" wave guide. The signal received after a path of $200 \mathrm{~mm}$ through global region corresponds to the output signal from the Global 1 region and serves as the input data for the local region modeled by FEM. This analysis takes place in the time domain. The second step consists in using the Global 1 output as two inputs (one for the S0 mode and the other for the A0 mode) in the FEM model. In order to avoid the reflections from the edges of the FEM domain, we extended the FEM domain outside the $100-\mathrm{mm}$ local region to a total length of $250 \mathrm{~mm}$ (Figure 4). The received signal from the local FEM model is the new input data for the Global 2 analytical region.

The hybrid global-local-global signal is compared with the analytical model, and the experimental results, received at R-PWAS after a path length of $565 \mathrm{~mm}$ (Figure 5). A good agreement for the S0 wave packet is observed. For the A0 mode, a good agreement is also observed but a slight time shift exists at the end of the A0 wave packet. We can conclude that this time domain HGL approach is in good agreement with the experimental results and with the analytical model. The slight difference in the A0 packet can be explained by the fact that these models, analytical and HGL, are 1D guided waves which are not exactly representative of the experimental results, i.e. 2D guided waves.

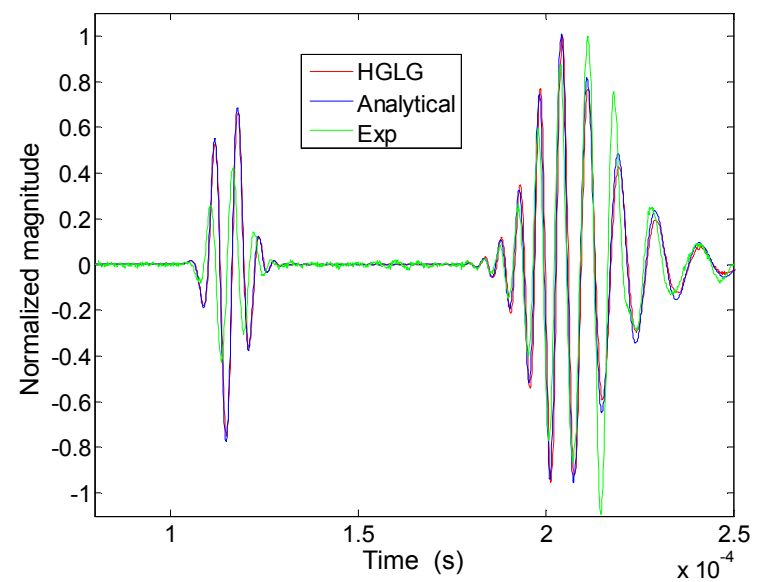

Figure 5: Comparison of the received signal after a distance travel of $565 \mathrm{~mm}$ for the analytical model, the experimental results and for the HGL three-step approach (global-localglobal). 


\section{Hybrid Global-Local approach for damage detection}

Metal structures exhibit a wide range of damage types including cracks and corrosion. The later may be uniform, pitting, galvanic, crevice, concentration cell, and graphite corrosion [33]. When a waveguide structure has changes in geometry, materials properties, supports, or attachments, the guided waves that propagate through it will be modified accordingly. E.g., loss of material due to corrosion presents geometrical changes which will cause the guided waves scattering and can be used for corrosion inspection. In this study, corrosion is simulated by a uniform notch in an aluminum plate. The notch depth and the width were increased gradually in order to simulate corrosion progression. This thickness loss produced a change in the waveguide elasto-dynamic impedance and thus caused (i) scattering and reflection; (ii) modification of the wave speed of the Lamb waves crossing the corrosion area; and (iii) mode conversion. In the HGL model, simulated corrosion was made on 3.2-mm thick aluminum plate. The location of the corrosion was on the middle of the local FEM area. Transient analysis of Lamb wave interaction with a surface notch in a plate is conducted using our HGL approach as described in the previous section. In practice, corrosion defects are geometrically complex and require multiple parameters to describe them and their scattering behaviors. We used the simplified shape of a uniform notch to reduce the number of parameters in order to better understand the changes causes by material loss.

Incident wave from the T-PWAS will propagate and then interact with the notch, undergoing mode conversion. Hence, there will be reflected waves traveling toward the left edge to be captured by TPWAS and transmitted waves traveling towards the right edge to be captured by the R-PWAS. If the incident wave is fixed, the reflection and transmission coefficients are affected by the severity of the structural discontinuity, which, in this case, is the notch geometry. These coefficients, when measured experimentally, can be used as a quantitative measure to characterize the discontinuity. To analyze the sensitivity of the Lamb waves to the size of the notch, the reflection coefficients of $\mathrm{A} 0$ and S0 from the notch are computed using our HGL approach. The rectangular notches have a constant width of $1 \mathrm{~mm}$ and depth varying from 1 to $3 \mathrm{~mm}$. The results are shown in Figure 6 for A0 input and in Figure 7 for S0 input. The reflection coefficients of A0 and S0 from a notch have been studied by Lowe et al. [34] and by Lowe and Diligent [35] by using an in-house FEM software. The results in Figure $6 \mathrm{a}$ and Figure $7 \mathrm{a}$ agree with their results. A simpler interpretation of the reflection coefficient curves against notch depth can be made here based on Figure 6 and Figure 7 by considering mode shape and energy conservation with respect to transmission, reflection, and mode conversion.

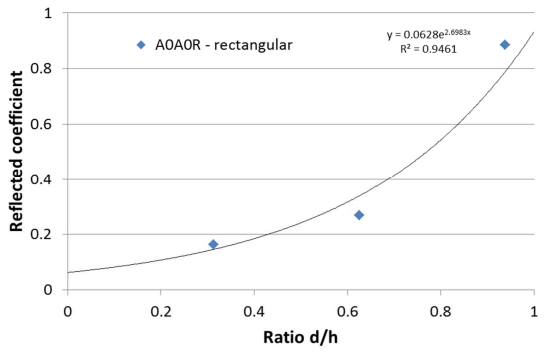

(a)



(b)



(c)

Figure 6: Coefficients versus normalized notch depth ratio for A0 incident wave packet; (a) reflected A0; (b) transmitted A0; (c) transmitted SO mode conversion. 
The case of A0 input is first discussed based on Figure 6. The notch depth is normalized by the plate thickness, $d / h$. As shown in Figure $6 \mathrm{a}$, for $d / h<0.6$, the notch depth is around $1 / 20$ the wavelength of A0 (which is $12.4 \mathrm{~mm}$ ). Hence, the wave will transmit through the notch almost completely with negligible reflection, which agrees well with the low coefficients obtained for the reflected A0 wave components. If the notch spans over the full depth, then the A0 wave is fully reflected with no conversion. The coefficient for the reflected A0 wave component approaches 1 and that for the trnasmitted S0 wave approaches 0 . In between these two extremes, the incident A0 wave will undergo mode conversion, partially transmitted and partially reflected containing both A0 and S0 components. It is also not surprising that in Figure $6 \mathrm{c}$, the transmitted S0 coefficient first increases and then decreases with depth, with the maximum value occurring when the notch depth is close to half the thickness of the plate.



(a)

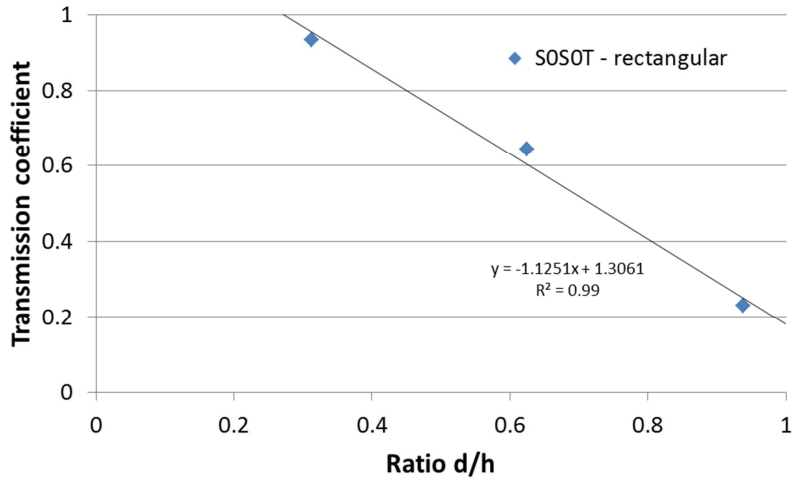

(b)

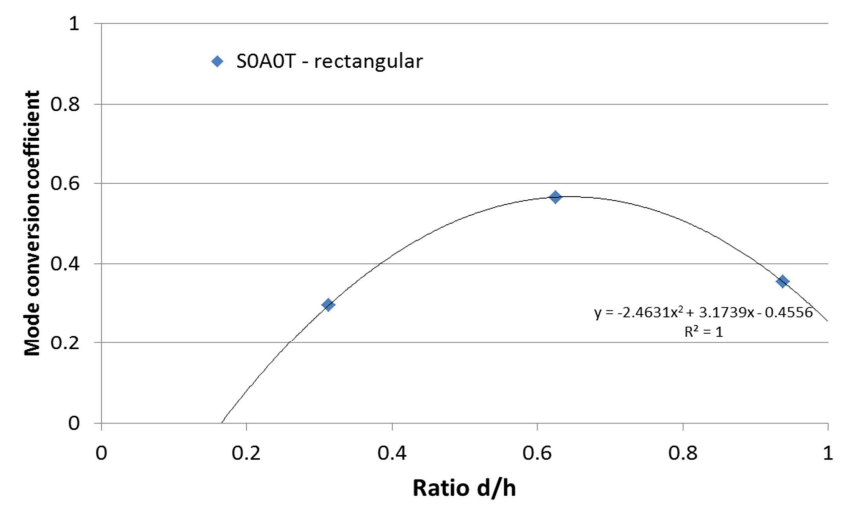

(c)

Figure 7: Coefficients versus normalized notch depth ratio for S0 incident wave packet. (a) reflectd S0; (b) tranmitted S0; (c) transmitted A0 mode conversion.

The reflection, transmission, and mode conversion coefficients for S0 input are plotted in Figure 7. A phenomenon similar to the A0 case can be observed where mode conversion is maximum when notch depth is about half plate thickness (Figure 7b). Unlike A0, the S0 exhibits good sensitivity even for a shallow notch. For $d / h>0.4$, the notch depth is around 1/100 wavelength of S0 (which is $35.9 \mathrm{~mm}$ ) and the reflection can be distinctly observed. This could be due to its small value at the surface meaning that any change becomes significant. The existence of surface crack destroys the symmetry of the wave, resulting in mode conversion. It is shown here that the mode shape also contributes significantly to the sensitivity of the detection. This is consistent with the observation by Ditri et al. [36] that energy distribution inside a plate for different Lamb modes could be used to predict their detectability due to the presence of cracks.

Similar analysis can be performed to evaluate the dependence of the coefficients on notch width, which for conciseness reasons will not be discussed in detail. They agree well with the results presented by Lowe et al. [34], and Lowe and Diligent [35]. The variation of the coefficients against notch width is somewhat periodic and relates to the wavenumber of the Lamb modes. 
A damage index can be computed by taking the ratio of the amplitudesd of the original and modeconverted waves as received at the R-PWAS. Figure 8 shows the damage index for S0 and for A0 input. For $d / h \leq 0.2$, the ratio based on the S0 input would be more sensitive than the A0 input mode. However, for $d / h>0.6$, the damage index is bigger for A0 mode input. So using the S0 and the A0 mode, we could quantify the dimension of the notch.

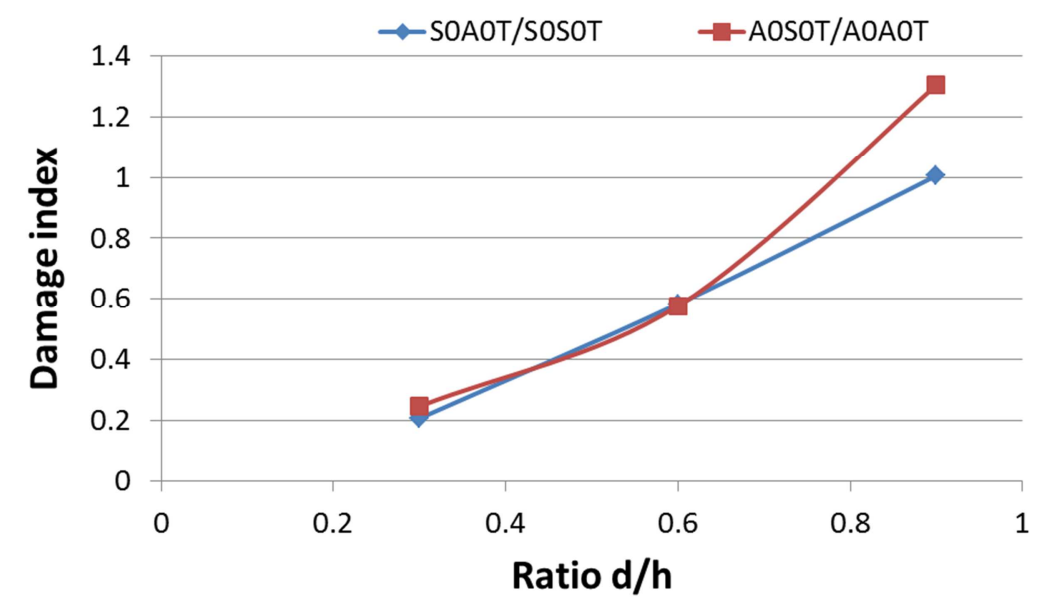

Figure 8: Damage index based on the ratio of the transmitted mode conversion and the undamaged transmitted for $\mathrm{SO}$ and for $\mathrm{AO}$ input.

\section{Conclusion}

This paper presents a hybrid finite element and analytical method to predict the 1-D guided wave propagation interaction with damage for nondestructive evaluation (NDE) and structural health monitoring (SHM) application. The finite element mesh is used to describe the region around the damage (defects or flaws). In contrast to other hybrid models developed elsewhere, the interaction between Lamb waves and defects is computed in the time domain using the explicit solver of the commercial finite element method (FEM) software ABAQUS. Compared to usual FEM model, the main advantage of the hybrid method is to reduce meaningfully the length of the mesh used to investigate the interaction of Lamb modes with defects and hence the computing time. Theory of guided wave propagation between two PWAS transducers was studied and an analytical model was built to give the theoretical waveforms of pitch-catch signals. Analytical modeling and finite element modeling have shown good match with experimental results, and can well describe guided wave propagation between two PWAS transducers. Lamb wave interaction with a notch is investigated by using this method, and the results obtained are consistent with respect to transmission, reflection and mode conversion. Because of the symmetric mode shape, S0 is more sensitive to the shallow notch than A0. By making use of the fact that the reflection increases with increase in notch depth and mode conversion are maximized when the notch is around half through the thickness of the plate, the reflection and conversion coefficients can be used to characterize the depth of the notch.

In future work, the analytical model will be extended to 3D circular PWAS analysis using Bessel function representation. Thus, we will realize the modeling of the guided wave propagation between two circular PWAS transducers in an arbitrary 2D geometry. The analytical modeling is expected to include damage/flaw in the plate structure, more complex structure (plate with double stiffener), and also include the second modes A1 and S1. The main complexity would be to develop a time domain hybrid global local model for 2-D wave propagation in structures.

\section{Acknowledgements}

This work was supported by the Office of Naval Research grant \#N00014-11-0271, program manager Dr. Ignacio Perez and by Air Force Office of Scientific Reasearch grant \#FA9550-11-10133, program manager Dr. David Stargel. 


\section{References}

[1] Grahn, T (2003) "Lamb wave scattering from a circular partly through-thickness hole in a plate", Wave Motion, 37(1), 63-80, 2003

[2] Castaings, M; Le Clezio, E; Hosten, B (2002) "Modal decomposition method for modeling the interaction of Lamb waves with cracks", The Journal of the Acoustical Society of America, 112(6), 2567-2582, 2002

[3] Diligent, O; Lowe, M J S; Cawley, P (2001) "Reflection and scattering of the S0 Lamb mode from circular defects in plates", AIP Conference Proceedings, 557(1), 134-141, 2001

[4] Alleyne, D N; Cawley, P (1992) "The interaction of Lamb waves with defects", Ultrasonics, Ferroelectrics and Frequency Control, IEEE Transactions on, 39(3), 381-397, 1992, doi: $10.1109 / 58.143172$

[5] Gresil, M; Lin, B; Shen, Y; Giurgiutiu, V (2011) "Predictive Modeling of Space Structures for SHM With PWAS Transducers," Proceedings of the ASME 2011 Conference on Smart Materials, Adaptive Structures and Intelligent Systems, Phoenix, Arizona, USA, 2011, pp. 525-534.

[6] Giurgiutiu, V; Gresil, M; Lin, B; Cuc, A; Shen, Y; Roman, C (2012) "Predictive modeling of piezoelectric wafer active sensors interaction with high-frequency structural waves and vibration", Acta Mechanica, 1-11, 2012, doi: 10.1007/s00707-012-0633-0

[7] Guo, N; Cawley, P (1993) "The interaction of Lamb waves with delaminations in composite laminates", J. Acoust. Soc. Am., 94(4), 2240-2246, 1993

[8] Galan, J M; Abascal, R (2005) "Boundary element solution for the bi-dimensional scattering of guided waves in laminate plates", Computers and Structures, 83(10-11), 740-757, 2005

[9] Peng, H; Meng, G; Li, F (2009) "Modeling of wave propagation in plate structures using three-dimensional spectral element method for damage detection", Journal of Sound and Vibration, 320(4-5), 942-954, 2009

[10] Gresil, M; Yu, L; Giurgiutiu, V (2011) "Fatigue crack detection in thick steel structures with piezoelectric wafer active sensors ", Proc. SPIE 7983, Nondestructive Characterization for Composite Materials, Aerospace Engineering, Civil Infrastructure, and Homeland Security, 7983 2Y, 2011, doi: doi:10.1117/12.882137.

[11] Goetschel, D B; Dong, S B; Muki, R (1982) "A Global Local Finite Element Analysis of Axisymmetric Scattering of Elastic Waves", Journal of Applied Mechanics, 49(4), 816-820, 1982

[12] Chang, Z; Mal, A K (1995) "A global local method for wave propagation across a lap joint", Numerical Methods in Structural Mechanics ASME, 204, 1-11, 1995

[13] Mal, A; Chang, Z (2000) "A semi-numerical method for elastic wave scattering calculations", Geophysical Journal International, 143, 328-334, 2000

[14] Hayashi, T (2004) "Guided wave animation using semi-analytical finite element method", 16th World Conference on NDT, 1328, 2004

[15] Hayashi, T; Kawashima, K; Sun, Z; Rose, J L (2003) "Analysis of flexural mode focusing by a semi-analytical finite element method", J. Acoust. Soc. Am., 113(3), 2003

[16] Hayashi, T; Murase, M; Song, W J; Park, I K (2006) "Development of Calculation Software for Guided Wave Propagation In a Pipe", AIP Conference Proceedings, 820(1), 173-180, 2006

[17] Bartoli, I; Lanza di Scalea, F; Fateh, M; Viola, E (2005) "Modeling guided wave propagation with application to the long-range defect detection in railroad tracks", NDT \& E International, 38(5), 325-334, 2005

[18] Sabra, K G; Srivastava, A; di Scalea, F L; Bartoli, I; Rizzo, P; Conti, S (2008) "Structural health monitoring by extraction of coherent guided waves from diffuse fields", The Journal of the Acoustical Society of America, 123(1), EL8-EL13, 2008

[19] Srivastava, A, "Quantitative structural health monitoring using ultrasonic guided waves," Structural Engineering, University of California, San Diego, 2009. 
[20] Viktorov, I A, Rayleigh and Lamb waves - Physical theory and application. New York Plenum Press, 1967.

[21] Auld, B A, Acoustic fields and waves in solids vol. I: Wiley Inter-sciences publication, 1973.

[22] Achenbach, J D; Xu, Y (1999) "Use of elasto-dynamic reciprocity to analyze point-load generated axisymmetric waves in plate", Wave Motion, 30(1), 57-67, 1999

[23] Rose, J L, Ultrasonic waves in solid media. Cambridge University: Cambridge University Press, 1999.

[24] http://www.me.sc.edu/Research/lamss/NV/html/L_software.html.

[25] Giurgiutiu, V, Structural Health Monitoring With Piezoelectric Wafer Active Sensor: Elsevier Academic Press, 2008.

[26] Gresil, M; Shen, Y; Giurgiutiu, V (2011) "Predictive modeling of ultrasonics SHM with PWAS transducers," 8th International Workshop on Structural Health Monitoring, Stanford, CA, USA, 2011.

[27] Gresil, M; Shen, Y; Giurgiutiu, V (2011) "Benchmark problems for predictive fem simulation of 1-D and 2-D guided waves for structural health monitoring with piezoelectric wafer active sensors", Review of Progress in Quantitative Non-destructive Evaluation, 1430(1), 18351842, 2011, doi: 10.1063/1.4716434

[28] Shen, Y; Giurgiutiu, V (2012) "Predictive simulation of nonlinear ultrasonics," Proc. SPIE, San-Diego, 2012.

[29] Shen, Y; Giurgiutiu, V (Submitted 2013) "WaveForm Revealer - An analytical predictive tool for the simulation of multi-mode guided waves interaction with damage," AIAA SDM, Boston, USA, Submitted 2013.

[30] Alleyne, D N; Cawley, P (1990) "A 2-dimensional Fourier transform method for the quantitative measurement of Lamb modes", IEEE Ultrasonics Symposium, 1143-1146, 1990

[31] Moser, F; Jacobs, L J; Qu, J (1999) "Modeling elastic wave propagation in waveguides with the finite element method", NDT \& E International, 32(4), 225-234, 1999

[32] ABAQUS, "Analysis User's Manual," 6-9.2 ed., 2008.

[33] Roberge, P, Corrosion inspection and monitoring. New-York: McGraw-Hill John Wiley \& Sons, 2007.

[34] Lowe, M J S; Cawley, P; Kao, J Y; Diligent, O (2002) "The low frequency reflection characteristics of the fundamental antisymmetric Lamb wave A0 from a rectangular notch in a plate", Journal of the Acoustical Society of America, 112, 2612-2622, 2002

[35] Lowe, M J S; Diligent, O (2002) "Low-frequency reflection characteristics of the S0 Lamb wave from a rectangular notch in plate", J. Acoust. Soc. Am., 111(1), 64-74, 2002

[36] Ditri, J J; Rose, J L; Chen, G (1992) "Mode selection criteria for defect detection optimization using Lamb waves", Review of Progress in Quantitative Non-destructive Evaluation, 11, 2109,1992 